\title{
Fluxon motion in long overlap and in-line Josephson junctions
}

\author{
Levring, O. A.; Pedersen, Niels Falsig; Samuelsen, Mogens Rugholm
}

Published in:

Applied Physics Letters

Link to article, DOI:

$10.1063 / 1.93251$

Publication date:

1982

Document Version

Publisher's PDF, also known as Version of record

Link back to DTU Orbit

Citation (APA):

Levring, O. A., Pedersen, N. F., \& Samuelsen, M. R. (1982). Fluxon motion in long overlap and in-line Josephson junctions. Applied Physics Letters, 40(9), 846-847. https://doi.org/10.1063/1.93251

\section{General rights}

Copyright and moral rights for the publications made accessible in the public portal are retained by the authors and/or other copyright owners and it is a condition of accessing publications that users recognise and abide by the legal requirements associated with these rights.

- Users may download and print one copy of any publication from the public portal for the purpose of private study or research.

- You may not further distribute the material or use it for any profit-making activity or commercial gain

- You may freely distribute the URL identifying the publication in the public portal

If you believe that this document breaches copyright please contact us providing details, and we will remove access to the work immediately and investigate your claim 
Hf alloys used as a component of the composite.

Part of this work was performed under the auspices of the Naval Ship Research and Development Center, Annapolis, Maryland, while $\mathrm{H}$. Wada was staying at NBS as a guest worker.

\footnotetext{
'K. Inoue and K. Tachikawa, Proc. 12th International Conf. Low. Temp.

Phys., Kyoto, 1970, p. 482.

${ }^{2}$ K. Inoue and K. Tachikawa, Appl. Phys. Lett. 25, 94 (1974).

${ }^{3} \mathrm{~K}$. Inoue and K. Tachikawa, Appl. Phys. Lett. 29, 386 (1976).

${ }^{4} \mathrm{~K}$. Inoue and K. Tachikawa, IEEE Trans. Magn. MAG-13, 840 (1977).

${ }^{5}$ First reported in this article.
}

${ }^{6} \mathrm{~K}$. Inoue, H. Wada, T. Kuroda, and K. Tachikawa, Appl. Phys. Lett. 38, 939 (1981).

${ }^{7}$ B. S. Brown, J. W. Hafstrom, and T. A. Klipert, J. Appl. Phys. 48, 1759 (1977).

${ }^{8}$ T. Luhman and M. Suenaga, IEEE Trans. Magn. MAG-13, 800 (1977).

${ }^{9}$ A. Pebler and E. A. Gulbransen, Trans. Met. Soc. AIME 239, 1593 (1967).

${ }^{10}$ E. A. Pushkarev, N. S. Petrenko, and V. A. Finkel, Phys. Status Solidi A 47, K145 (1978).

"J. W. Ekin, Adv. Cryo. Eng. 24, 306 (1978)

${ }^{12} \mathrm{~J}$. W. Ekin, Cryogenics 20,611 (1980).

${ }^{13}$ J. W. Ekin, IEEE Trans. Magn. MAG-15, 197 (1979).

${ }^{14} \mathrm{G}$. Rupp, IEEE Trans. Magn. MAG-15, 189 (1979).

${ }^{15}$ See, for example, K. Togano, T. Asano, and K. Tachikawa, J. Less Comm. Metals 68, 15 (1979); K. Tachikawa, Y. Tanaka, Y. Yoshida, T. Asano, and Y. Iwasa, IEEE Trans. Magn. MAG-15, 391 (1979).

\title{
Fluxon motion in long overlap and inline Josephson junctions
}

\author{
O. A. Levring, N. F. Pedersen, and M. R. Samuelsen \\ Physics Laboratory I, The Technical University of Denmark, DK-2800 Lyngby, Denmark
}

(Received 4 February 1982; accepted for publication 16 February 1982)

\begin{abstract}
The motion of a single fluxon in long Josephson junctions of the overlap and inline geometries is investigated. It is concluded that if the junction is long and the damping is not too large then zerofield steps exist also in the inline junction. These zero-field steps are found to be mathematically identical to those of the overlap junctions in spite of the fact that the fluxon dynamics are quite different in the two cases.
\end{abstract}

PACS numbers: $74.50 .+\mathrm{r}, 84.40 . \mathrm{Mk}, 85.25 .+\mathrm{k}$

Recently large interest has been focused on fluxon motion in long Josephson junctions. Two different geometries are usually considered. In the overlap type of junctions the so-called zero-field steps are observed in the $I V$ curve. They are due to resonant motion of (a few) fluxons being reflected at the boundaries. ${ }^{1,2}$ In the inline geometry a so-called resistive branch may be observed in the $I V$ characteristic. ${ }^{3} T$ his is due to a (continuous) generation of fluxons in one end and antifluxons in the other end of the junction and a subsequent annihilation in the center. ${ }^{4}$ In this letter we discuss the differences and similarities between fluxon motion in the two geometries. One of the important similarities is that zero-field steps may also exist in inline junctions if certain conditions are satisfied.

The motion of fluxon in a long and narrow Josephson junction is governed by the perturbed sine-Gordon equation $^{5}$

$$
\phi_{x x}-\phi_{t t}=\sin \phi+\alpha \phi_{t}+\eta,
$$

where $\phi$ is phase difference between the two superconducing films. The spatial variable in the long direction, $x$, is measured in units of the Josephson penetration depth $\lambda_{\text {, }}$ $=\left(\hbar / 2 e d \mu_{0} J\right)^{1 / 2}$, and time $t$ in units of the reciprocal plasma frequency $\omega_{0}^{-1}$, where $\omega_{0}=(2 e J / \hbar C)^{1 / 2}$. Here $J$ is the Josephson current density, $d$ is the magnetic thickness of the barrier, and $C$ is the capacitance per unit area. $\alpha=1 / \sqrt{ } \beta_{c}$, where $\beta_{c}$ is the McCumber parameter $\beta_{c}=2 e J C / \hbar G^{2}$, where $G$ is the conductance per unit area due to quasiparticles. The unnormalized length of the junction is $L$ (defines the $x$ direction) and the unnormalized width is $W$. These dimensions are subject to the conditions $L>\lambda_{J}>W$. The surface current density in the London penetration layer of one of the films is given by

$$
i_{x}=\lambda_{s} J \phi_{x}
$$

and thus the magnetic field in the oxide layer is

$$
H_{y}=i_{x}=\lambda_{J} J \phi_{x} \text {. }
$$

The above considerations are common to the overlap and inline geometries; we now introduce the bias current $I_{\mathrm{dc}}$ and boundary conditions separately for the two cases.

In the overlap junction [Fig. 1(a)] the bias current is uniformly distributed across the long direction of the junction and enters through $\eta$ in Eq. (1), which is then given by

$$
\eta^{\mathrm{ov}}=I_{\mathrm{dc}} / J W L \text {. }
$$

Defining $\kappa$ as the magnitude of the (normalized) magnetic field [Eq. (3)] at the junction ends the boundary conditions are

$$
\kappa^{\mathrm{ov}}=\phi_{x}(0, t)=\phi_{x}(l, t)=0,
$$
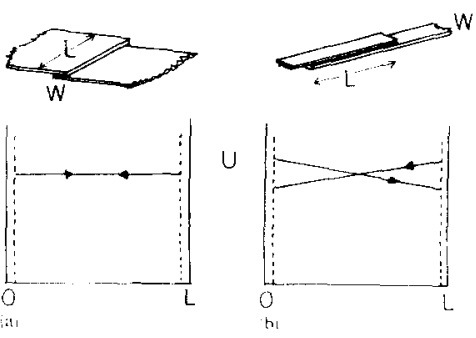

FIG. 1. (a) Overlap geometry. The fluxon propagates with a constant velocity defined by loss and bias. (b) Inline geometry. The fluxon receives energy when being reflected at the ends and loses energy when propagating along the line due to losses. 
where $l$ is the normalized length, $l=L / \lambda_{J}(l>1)$. Here we note that because of the uniformly distributed bias current the critical current of the junction $I_{c}^{\mathrm{ov}}$ is given by $I_{c}^{\mathrm{ov}}=W L J$.

In the inline junction [Fig. 1(b)] we have

$$
\eta^{\text {in }}=0
$$

in Eq. (1), and the bias current is introduced through the magnetic field it creates at the ends of the junction through the boundary conditions

$$
\phi_{x}(0, t)=-\phi_{x}(l, t)=I_{\mathrm{dc}} / 2 \lambda_{J} W J=\kappa^{\mathrm{in}} .
$$

For this case the critical current $I_{c}^{\mathrm{in}}$ is given by $I_{c}^{\mathrm{in}}=4 \lambda_{J} W J$, which is smaller than in the overlap case.

If (for both geometries) one fluxon moves with average (normalized) velocity $u$, a dc voltage $V_{\text {dc }}$ (normalized to $\left.\hbar \omega_{0} / 2 e\right)$ is developed across the junction. Here $V_{\mathrm{dc}}$ $=4 \pi u / 2 l=4 \pi / T$, where $T$ is the (normalized) period. In order to determine this average velocity we write the total (normalized) energy on the Josephson transmission line ${ }^{5}$

$H=\int_{i}^{l}\left(\frac{1}{2} \phi_{x}^{2}+\frac{1}{2} \phi_{t}^{2}+(1-\cos \phi)\right) d x$.

Differentiating Eq. (8) with respect to time and using $\eta$ and $\kappa$ (for the particular geometry) we obtain the perturbation result

$\frac{d H}{d t}=-\kappa\left[\phi_{t}(l, t)+\phi_{t}(0, t)\right]-8 u^{2} \gamma(u) \alpha+2 \pi \eta u$.

To obtain the last two terms we have used the single-soliton solution to the pure sine-Gordon equation. $\gamma(u)$ is the Lorentz factor $\gamma(u)=\left(1-u^{2}\right)^{-1 / 2}$. In a stationary condition $d H / d t$ integrated over one period should be zero. Since the phase change over one period is $-4 \pi$ we obtain from $\mathrm{Eq}$. (9)

$$
\alpha 8 u^{2} \gamma(u)=2 \pi \eta u+\kappa 8 \pi / T .
$$

Since $\kappa=0$ in the overlap geometry, the velocity $u$ is constant. Physically the fluxon is maintained at this constant velocity through the Lorentz force from the uniformly distributed bias current [the term $\eta$ in Eq. (10)]. This is shown in Fig. 1(a).

For the inline geometry $\eta=0$, and the soliton velocity decreases along the Josephson line [Fig. 1(b)]. When it reaches the boundary it gets an energy input of $4 \pi \kappa$ from the boundary condition and continues its steady-state motion. It should be noted that for these events to occur as described, it is required that the soliton reaches the other end of the junction with only a small velocity change, i.e. $\alpha l<1$ or $l<V \beta_{c}$.

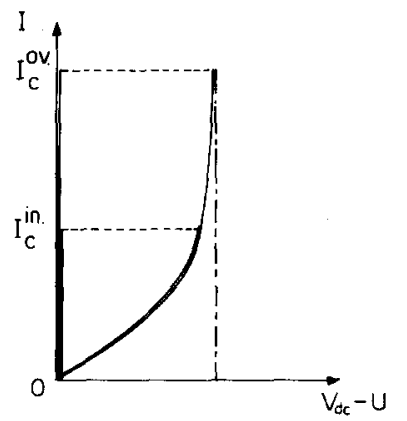

FIG. 2. Supercurrent and first zerofield step for both overlap and inline geometry. The only difference is that due to self-field effects, the critical supercurrent for an inline junction is smaller than the critical supercurrent for the overlap junction.
The (normalized) voltage across the junction, $V_{\mathrm{dc}}$, is given by $V_{\mathrm{dc}}=2 \pi u / l$, where $u$ is determined from Eq. (10).

$u \gamma(u)= \begin{cases}\frac{\pi \eta}{4 \alpha}=\frac{\pi I_{\mathrm{dc}}}{4 \alpha J W L} & \text { (overlap) } \\ \frac{\pi \kappa}{2 \alpha l}=\frac{\pi I_{\mathrm{dc}}}{4 \alpha J W L} & \text { (inline) }\end{cases}$

Hence, the results for the two geometries are identical and the so-called zero-field steps from overlap junction also exist in inline junction as long as $l<\sqrt{ } \beta_{c}$.

The common result for the two geometries is shown in Fig. 2. The main difference is that the critical current for the inline junction $I_{c}^{\mathrm{in}}$ is smaller than that for the overlap junction $I_{c}^{\text {ov }}$, as discussed earlier. Higher-order zero-field steps may be obtained by scaling the voltage with the number of fluxons. If, in the inline geometry, $\gg \vee \beta_{\mathrm{c}}$ and $I_{\mathrm{dc}}>I_{\mathrm{c}}^{\text {in }}$ fluxons are continuously created in one end of the junction and antifluxons in the other. They annihilate each other in the center of the junction and give rise to the so-called displaced linear branch as discussed by Scott and Johnson. ${ }^{4}$

In conclusion, we note that for inline junction with $l \ll V \beta_{c}$ and $I_{\mathrm{dc}}<I_{c}^{\text {in }}$ zero-field steps obeying exactly the same equation as those of overlap junctions occur. This happens in spite of the fact that the soliton dynamics are quite different in the two cases. Because of the more restricted current range for the inline junctions, experimentally observed singularities in the $I V$ curve may erroneously be interpreted as different from those found in overlap junctions.

'T. A. Fulton and R. C. Dynes, Solid State Commun. 12, 57 (1973).

${ }^{2}$ R. D. Parmentier, in Solitons in Action, edited by K. Lonngren and A. Scott (Academic, New York, 1978), pp. 173-199.

${ }^{3}$ A. Barone, J. Appl. Phys. 42, 2747 (1971).

${ }^{4}$ A. C. Scott and W. J. Johnson, Appl. Phys. Lett. 14, 316 (1969).

${ }^{5}$ A. C. Scott and D. W. McLaughlin, Phys. Rev. A 18, 1652 (1978). 